\title{
Obscure gastrointestinal bleeding: difficulties in comparing CT enterography and video capsule endoscopy
}

\author{
Philippe Soyer
}

Received: 15 December 2011 / Accepted: 16 January 2012 / Published online: 24 March 2012

(C) European Society of Radiology 2012

\begin{abstract}
A paper reports the results of a retrospective study that was designed to evaluate the potential role of video capsule endoscopy (VCE) in elucidating the cause of bleeding in patients with obscure gastrointestinal bleeding (OGIB) for whom CT enterography was negative. The authors highlight the limitations of dual-phase CT enterography for the detection of flat lesions of the small bowel such as ulcers, angiodysplasias or arteriovenous malformations, and confirm the superiority of VCE for the detection of this category of lesions. This commentary discusses some of the issues raised. Key Points

- Video capsule endoscopy surpasses CT enterography in detecting flat small bowel lesions.

- Retrospective VCE and CT enterography findings in obscure bleeding need further evaluation.

- A fair and unbiased comparison of the two investigations is still needed.
\end{abstract}

\footnotetext{
P. Soyer $(\bowtie)$

Department of Imaging \& Nuclear Medicine,

Hôpital Lariboisière-APHP 2,

2 rue Ambroise Paré,

75010 Paris, France

e-mail: philippe.soyer@1rb.aphp.fr

P. Soyer

UMR INSERM 965-Paris 7 "Angiogenèse

et recherche translationnelle",

2 rue Amboise Paré,

75010 Paris, France

P. Soyer

Université Diderot-Paris 7,

10 Avenue de Verdun,

75010 Paris, France
}

Heo et al. present the results of a retrospective study that was designed to evaluate the potential role of video capsule endoscopy (VCE) in elucidating the cause of bleeding in patients with obscure gastrointestinal bleeding (OGIB) for whom CT enterography was negative [1]. The authors performed an interesting study involving 30 patients with negative CT enterography who had further VCE. A definite diagnosis was made for 17 patients $(17 / 30 ; 57 \%)$ owing to the findings at VCE and, overall, $23 \%$ of patients received specific treatment on the basis of VCE findings. Their results highlight the limitations of dual-phase CT enterography for the detection of a specific category of small bowel lesions and confirm the superiority of VCE for the detection of the same category of lesions. Heo et al. have designed their study in this way because at their institution CT enterography is the initial investigation in patients with OGIB and their study design is directly derived from their actual algorithmic approach [1].

Using a reversed algorithm, Agrawal et al. recently found similar results for CT enterography in terms of detection of small bowel lesions [2]. They used CT enterography in patients with OGIB for whom VCE was negative. Using dual phase CT enterography, they did not detect the cause of bleeding in any of the 11 patients with occult OGIB and negative VCE [2].

The results of these two studies can be interpreted in two different ways. One way should be to definitely consider that CT enterography has limited or even no value for the evaluation of patients with OGIB and that VCE should be the favoured test for this indication. The alternative way would be to admit that these two techniques are complementary and not competing tests for this elective indication. I must admit that I strongly agree with the second approach for several reasons that I will discuss herein.

Because of their study design with inclusion of patients with negative CT enterography, Heo et al. did not address the limitations of VCE [1]. In this regard, none of the patients in 
the study by Heo et al. had small bowel tumours [1]. I assume that this is because they were detected with CT enterography, so that VCE was not needed. Fortunately, other studies have highlighted the limitations of VCE for this elective indication. It has been admitted that detection of a small bowel tumour is the area in which VCE has the poorest performance. One reason is that some small bowel tumours are of submucosal origin and produce little mass effect or displacement so that they are hardly visible at VCE [3]. Postgate et al. reported five cases of small bowel tumours missed at VCE [4]. Ross et al. reported false-negative findings at VCE in 10 out of 15 patients $(67 \%)$ with small bowel tumours [5]. Baichi et al. missed three small-bowel tumours out of a series of ten with VCE [6]. The rate of false-negative findings may reach up to $19 \%$ for VCE in the detection of small bowel tumours [7, 8]. This is because VCE shows the mucosal layer only and may not detect submucosal tumours such as stromal tumours, leiomyosarcomas, Kaposi's sarcoma, and metastases from lung cancer, breast cancer and melanoma [9].

Early studies in the gastroenterological literature suggested that VCE might play a potential role in the detection of small bowel tumours. However, as experience accumulates, the limitations of this technique are becoming increasingly evident and recent reports suggest that the detection of small bowel tumours may not be the preferred indication $[4,10]$. When a small bowel tumour is suspected on VCE, it has to be further confirmed and characterised by $\mathrm{CT}$ in conjunction with enterography or enteroclysis $[10,11]$. Conversely, in the case of a suspected small bowel tumour, negative VCE findings have to be confirmed by one of these imaging investigations [10, 11]. This strongly questions the use of VCE for this indication, and suggests that $\mathrm{CT}$ enterography or preferably $\mathrm{CT}$ enteroclysis should be favoured.

Heo et al. have conducted a nice study that provides a useful conclusion [1]. However, the study design is somewhat unfair from a radiologist's point of view. In their study, patients were retrospectively included when they had negative results at CT enterography, so that there was no direct comparison between VCE and CT enterography, and the respective values of the two techniques in this specific situation were not analysed. Using a larger spectrum of patient population (i.e. those who had both CT enterography and VCE, irrespective of the result of the first test performed) the authors would have made a more meaningful study.

In the study by Heo et al., VCE findings were classified as highly relevant with a high bleeding potential (P2), moderately relevant with an intermediate bleeding potential (P1) or normal (P0), according to an accepted standard practice [12]. In many comparative studies, VCE was the test being evaluated but it also served for some patients as the standard of reference, which introduces major flaws. In most published studies, because not all patients received a confirmatory endoscopic test or had surgery and pathological confirmation, a substantial proportion of positive findings at VCE was not fully confirmed and results were often reported in terms of "diagnostic yield". In this regard, Haghighi et al. have reported interesting results involving 160 patients with OGIB in whom angioectasias, erosions and ulcers were found in $68 \%, 29 \%$ and $21 \%$ of patients at VCE, supporting the evidence that this test has a high diagnostic yield [13]. However, the same authors have found similar or even greater proportions of small bowel abnormalities in healthy patients who served as controls [13]. It has become evident that Haghighi et al. have pointed out a critical issue, which is the ability to distinguish between an actual source of bleeding that needs further therapy, a potential source of bleeding or even an incidental finding [13].

Another concern raised by the study by Heo et al. is the validity of the standard of reference [1]. Although I must admit that they used a well-accepted standard, one critical point must be addressed. Like many other researchers who compared VCE with other tests, Heo et al. used the intention to treat as a reference test in many cases instead of confirmation of VCE findings with other robust tests (i.e. pathological analysis, double balloon enteroscopy or surgical findings). Consequently, this approach is likely to be biased because it was not possible to make sure that all positive findings at VCE were actually true-positive ones. It may be argued that this approach favourably overestimated the actual sensitivity of VCE at the penalty of CT enterography.

One undisputed advantage of CT enterography or CT enteroclysis over VCE is the ability to detect extra-enteric abnormalities $[9,14]$. Some of them (such as the presence of hepatic metastases in the case of malignant small-bowel tumours) may have a major impact on the therapeutic approach [9], whereas others (such as the detection of mesenteric mass with focal calcification and surrounding desmoplastic reaction) may be a clue to a specific diagnosis [14]. In addition, CT enterography may show incidental findings in up to $20 \%$ of patients, and a proportion may require additional investigations or alteration of the therapeutic decision $[2,15]$.

Another issue that needs further investigation is the occurrence of false-positive findings at VCE. Although critical, this issue, to my knowledge, has rarely been addressed in the literature. My colleagues and I have recently published a study that compared VCE and $\mathrm{CT}$ enteroclysis in patients with OGIB [11]. In our series, which included 32 patients, CT enteroclysis definitely excluded three suspected tumours in three different patients because of bulges at VCE that were misinterpreted as possible tumours [11]. False-positive findings at VCE were also recently reported by Hara et al. in two patients who were erroneously considered as having small bowel lipomas [16].

Besides the above-mentioned limitations of VCE, another, which is of major importance, is VCE retention. A retrospective study from Sweden involving 2,300 examinations reported VCE retention in 31 patients $(1.3 \%)$ and 27 of them required 
surgery for VCE removal. More importantly, two patients died after surgery [17]. I must say that patients with OGIB are considered to be at low risk of VCE retention, so that this frequency observed in a more general population should be lower in patients with OGIB [18]. Finally, in the same study, $20 \%$ of VCE examinations were incomplete, with VCE not reaching the caecum during the recording time [17]. Incompleteness may be a matter of concern, especially when it is important to exclude small bowel disease.

Although VCE has a high diagnostic yield in patients with overt OGIB, endoscopists commonly acknowledge that underlying lesions may be obscured by blood so that more precise characterisation of causative abnormality must be obtained by CT enterography. In this regard, although Agrawal et al. were not able to detect the cause of bleeding in any of the 11 patients with occult OGIB using CT enterography, they reported that CT enterography depicted the cause of bleeding in $7 / 14$ patients $(50 \%)$ with overt OGIB for whom VCE was inconclusive [2]. These results suggest a major role for CT enterography in overt OGIB, should the patient be haemodynamically stable, and confirm that $\mathrm{CT}$ shows better performances when OGIB is overt $[19,20]$.

To be fair, I have to mention that $\mathrm{CT}$ enterography has major limitations in the evaluation of OGIB. This is because vascular abnormalities account for $80 \%$ of OGIB and especially angiodysplasia, which accounts for $50-60 \%$ of OGIB, whereas small bowel tumours are found in only $1.6 \%$ to $2.4 \%$ of patients undergoing VCE $[7,8]$. VCE allows direct visualisation of the small bowel mucosa and has a high sensitivity for the detection of flat lesions such as ulcers, angiodysplasias or arteriovenous malformations, which are lesions beyond the reach of the best $\mathrm{CT}$ resolution available. In my experience, VCE shows ulcers and angioectasias that are not visible at CT enteroclysis [11]. A large number of papers have showed that among the variable causes of OGIB, the one most commonly missed at CT enterography was small-bowel angioectasia.

Other questions are emerging from the most recent studies $[16,20]$. Of these, it is important to know to what extent the use of multiple image acquisitions during different phases might improve the diagnostic capabilities of CT enterography. This is a critical issue because multiple phases markedly increase the radiation dose given to the patient. Using a triphasic technique including unenhanced, arterial and enteric phases, Lee et al. were able to detect the cause of OGIB in $16 / 65$ patients (25\%); of these, the bleeding lesion was seen during the arterial phase only in 3 patients [20]. Also using the triphasic $\mathrm{CT}$ enterography technique, Hara et al. reported more limited additional value for multiple phases [16]. These researchers found a sensitivity of $22 \%(2 / 9)$ for CT enterography in patients with OGIB [16]. In addition, Hara et al. showed poor detection for mucosal lesions such as ulcers and vascular malformations, as is the case using a single acquisition technique [16]. One must admit that, at this time, a high radiation dose is not so valuable. However, these results were obtained from small sample sizes so that more studies have to be done to fully consider the potential benefit of multiple phase acquisition.

Another question that warrants further investigation is the better diagnostic performance of CT enteroclysis compared with CT enterography. At many centres CT enterography is used instead of CT enteroclysis because it is better tolerated by the patient and performed more rapidly. However, in my opinion, this argument is not acceptable. Patients with OGIB should benefit from the best test available and not from the most acceptable one. Should the same test combine both qualities, it can thus be selected. Conversely, if such a test does not exist, I believe that the most accurate one should be favoured. At this time there is no crystal-clear answer to that question, but this should stimulate further studies.

Although the study by Heo et al. reported interesting results, many questions remain unanswered [1]. Of these, the most important one is which is the best test depending on pre-test probability. In other words, is there a specific group of patients with OGIB in whom one test may be superior to the other. What is clear to me now, and this is supported by the existing literature, is that CT enterography is better than VCE for the detection of small bowel tumours $[11,21]$. Accordingly, the pretest probability of a specific cause of OGIB should be considered when selecting the first-line test. In this regard, in patients less than 50 years of age, small bowel tumour is the most frequent cause of OGIB $[9,21]$. Consequently, CT enterography should be the favoured test in this specific age category of patients. Similarly, when OGIB is overt, it is reasonable to perform CT enterography first, and when CT enterography is negative, to consider VCE as a second-line examination, as has been nicely shown by Heo et al. [1]. To go further in the right direction indicated by Heo et al., the design of the study by Lee et al. should serve as a template for future studies [20]. These authors used a multivariate analysis to find if any independent variables were significantly associated with a high diagnostic yield of CT enterography [20]. In my opinion, future comparative studies between diagnostic tests in OGIB should be done more comprehensively, with respect to the clinical situation, the patient's specificity and the impact on therapy using multiple regression models.

Currently, two different diagnostic approaches to OGIB exist. The first is based on the use of CT enterography as an initial test only in patients with contraindications for VCE and in those with suspected small bowel stricture or tumour [22]. The second suggests that the role of CT enterography may be expanded to a first-line test in a broader population of patients. It is clear that $\mathrm{CT}$ enterography or $\mathrm{CT}$ enteroclysis and VCE are not competing but complementary techniques, the limitations of one being overcome by the advantages of the other. Results from the most recent literature suggest a complementary role 
for CT enterography or CT enteroclysis and VCE in the evaluation of patients with OGIB $[3,11]$. However, because economic pressures favour the use of less expensive strategies, the two tests will not be performed routinely in the same patient. The question lies in deciding when one of these two approaches is the most appropriate.

As a conclusion, I advocate further studies on CT enterography and CT enteroclysis in patients with occult OGIB, with direct comparisons with VCE, and newer enteroscopy techniques, such as double balloon and spiral enteroscopy, and valid confirmation of the positive and negative findings. The question that remains unanswered is to determine to what extent CT enterography or CT enteroclysis and VCE are complementary and not competing techniques. Although some answers can be found in the literature published to date, this should be further evaluated by outcome-based, unbiased, large and well-designed prospective studies.

\section{References}

1. Heo HM, Park CH, Lim JS et al (2012) The role of capsule endoscopy after negative $\mathrm{CT}$ enterography in patients with obscure gastrointestinal bleeding. Eur Radiol. doi:10.1007/s00330-011-2374-1

2. Agrawal JR, Travis AC, Mortele KJ et al (2011) Diagnostic yield of dual-phase CT enterography in patients with obscure gastrointestinal bleeding and a nondiagnostic capsule endoscopy. J Gastroenterol Hepatol. doi:10.1111/j.1440-1746.2011.06959.x

3. Zhang BL, Jiang LL, Chen CX, Zhong BS, Li YM (2010) Diagnosis of obscure gastrointestinal hemorrhage with capsule endoscopy in combination with multiple-detector computed tomography. J Gastroenterol Hepatol 25:75-79

4. Postgate A, Despott E, Burling D et al (2008) Significant smallbowel lesions detected by alternative diagnostic modalities after negative capsule endoscopy. Gastrointest Endosc 68:1209-1214

5. Ross A, Mehdizadeh S, Tokar J et al (2008) Double balloon enteroscopy detects small bowel mass lesions missed by capsule endoscopy. Dig Dis Sci 53:2140-2143

6. Baichi M, Arifuddin RM, Mantry PS (2007) Small-bowel masses found and missed on capsule endoscopy for obscure bleeding. Scand J Gastroenterol 42:1127-1132

7. Lewis BS, Eisen GM, Friedman S (2005) A pooled analysis to evaluate results of capsule endoscopy trials. Endoscopy 37:960-965

8. Pennazio M, Rondonotti E, de Franchis R (2008) Capsule endoscopy in neoplastic diseases. World J Gastroenterol 14:5245-5253

9. Soyer P, Boudiaf M, Fishman EK, Hoeffel C, Dray X, Manfredi R et al (2011) Imaging of malignant neoplasms of the mesenteric small bowel: new trends and perspectives. Crit Rev Oncol Hematol 80:10-30

10. Maglinte DT (2005) Capsule imaging and the role of radiology in the investigation of diseases of the small bowel. Radiology 236:763-767

11. Khalife S, Soyer P, Alatawi A et al (2011) Obscure gastrointestinal bleeding: preliminary comparison of 64 -section CT enteroclysis with video capsule endoscopy. Eur Radiol 21:79-86

12. Saurin JC, Delvaux M, Gaudin JL et al (2003) Diagnostic value of endoscopic capsule in patients with obscure digestive bleeding: blinded comparison with video push-enteroscopy. Endoscopy $35: 576-584$
13. Haghighi D, Zuccaro G, Vargo J et al (2005) Comparison of capsule endoscopy (CE) findings of healthy subjects (HS) to an obscure gastrointestinal bleeding (OGIB) patient population. Gastrointest Endosc 61:AB 104

14. Kamaoui I, De-Luca V, Ficarelli S, Mennesson N, LombardBohas C, Pilleul F (2010) Value of CT enteroclysis in suspected small-bowel carcinoid tumors. AJR Am J Roentgenol 194:629633

15. Soyer P, Boudiaf M, Martin-Grivaud S et al (2009) Abdominopelvic extra-enteric findings at computed tomography enteroclysis: a retrospective evaluation of 430 consecutive patients. Clin Imaging 33:188-195

16. Hara AK, Walker FB, Silva AC, Leighton JA (2009) Preliminary estimate of triphasic CT enterography performance in hemodynamically stable patients with suspected gastrointestinal bleeding. Am J Roentgenol 193:1252-1260

17. Höög CM, Bark LÅ, Arkani J, Gorsetman J, Broström O, Sjöqvist U (2012) Capsule retentions and incomplete capsule endoscopy examinations: an analysis of 2300 examinations. Gastroenterol Res Pract 2012:518718

18. Cave D, Legnani P, de Franchis R, Lewis BS (2005) ICCE consensus for capsule retention. Endoscopy 37:1065-1067

19. Duchat F, Soyer P, Boudiaf M et al (2010) Multi-detector row CT of patients with acute intestinal bleeding: a new perspective using multiplanar and MIP reformations from submillimeter isotropic voxels. Abdom Imaging 35:296-305

20. Lee SS, Oh TS, Kim HJ et al (2011) Obscure gastrointestinal bleeding: diagnostic performance of multidetector CT enterography. Radiology 259:739-748

21. Filippone A, Cianci R, Milano A, Valeriano S, Di Mizio V, Storto ML (2008) Obscure gastrointestinal bleeding and small bowel pathology: comparison between wireless capsule endoscopy and multidetector-row CT enteroclysis. Abdom Imaging 33:398406

22. Pasha SF, Hara AK, Leighton JA (2009) Diagnostic evaluation and management of obscure gastrointestinal bleeding: a changing paradigm. Gastroenterol Hepatol (N Y) 5:839-850

\section{Authors' Reply}

Sung Pil Hong, MD, PhD

Department of Internal Medicine and Institute of Gastroenterology Yonsei University College of Medicine, Seoul, Korea

On behalf of Heo HM, Park CH, Lim JS, Lee JH, Kim BK, Cheon JH, Kim TI, Kim WH, Hong SP.

We thoroughly appreciate the above commentary with regard to our original publication, which was designed to evaluate the potential role of capsule endoscopy in elucidating small bowel lesions in patients with obscure gastrointestinal bleeding (OGIB) after negative CT enterography. The commentary describes well the limitations of capsule endoscopy and the advantages of CT enterography in evaluating the patients with OGIB.

At present, most of the literature recommends capsule endoscopy as a first-line diagnostic method in evaluating patients with OGIB after a negative bidirectional endoscopy because of low invasiveness, no need for sedation and direct visualisation of the small bowel [1]. CT enterography is recommended when capsule endoscopy is not indicated in cases with suspected small bowel strictures. However, we fully agree that it is time to expand the indications for CT enterography in evaluating patients with OGIB. From this viewpoint, it is interesting to suggest, as proposed in the commentary, that $\mathrm{CT}$ enterography be 
recommended as a first-line diagnostic test in patients younger than 50 years, considering the limitation of capsule endoscopy in evaluating small bowel tumours. However, this effort should be based on the evidence. There have been no studies to elucidate the advantages of CT enterography in patients with overt OGIB compared with capsule endoscopy. Indeed, overt bleeding is a definite condition to increase the diagnostic yield of capsule endoscopy in patients with OGIB [2].

The commentary also mentions, as another advantage, that CT enterography is able to detect extra-enteric abnormalities in up to $60 \%$ [3]. However, more than $50 \%$ of patients presented negligible abnormalities in that study. In CT colonography, only $4.5 \%$ have significant extra-colonic abnormalities [4]. These findings clearly suggest that a considerable number of patients with positive extra-enteric findings upon $\mathrm{CT}$ enterography may receive further unnecessary investigations.

We totally agree that capsule endoscopy and CT enterography are complementary tests in patients with OGIB. The present publication puts forth only one recommendation, namely that capsule endoscopy should be performed to elucidate the bleeding sources for the small bowel in patients with OGIB after negative CT enterography. However, a lot of questions still remain unanswered. Accordingly, the evaluation of small bowel lesions in patients with OGIB continues to present great challenges, which further research should clarify.

\section{References}

1. Rockey DC (2010) Occult and obscure gastrointestinal bleeding: causes and clinical management. Nature reviews. Gastroenterol Hepatol 7:265-279

2. Pennazio M, Santucci R, Rondonotti E, et al (2004) Outcome of patients with obscure gastrointestinal bleeding after capsule endoscopy: report of 100 consecutive cases. Gastroenterology 126:643-653

3. Soyer P, Boudiaf M, Martin Grivaud S, et al (2009) Abdominopelvic extra-enteric findings at computed tomography enteroclysis: a retrospective evaluation of 430 consecutive patients. Clin Imaging 33:188195

\section{Editors note:}

Further discussion on this topic should be sent to the Opinions, Comments, Controversies (Opinions) section on the European Radiology www.european-radiology.org 